\title{
L'explication du relief de côtes de la France de l'Est dans la première moitié du XXe siècle
}

The explanation of the scarplands of eastern France in the first half of XXth century

Die Erklärung der Schichtstufenlandentwicklung in Ostfrankreich während der ersten Hälfte des XX. Jhs

\section{Michel Deshaies}

\section{OpenEdition}

\section{Journals}

Édition électronique

URL : http://journals.openedition.org/rge/4508

DOI : $10.4000 /$ rge.4508

ISSN : 2108-6478

\section{Éditeur}

Association des géographes de l'Est

Édition imprimée

Date de publication : 1 janvier 1999

ISSN : 0035-3213

\section{Référence électronique}

Michel Deshaies, «L'explication du relief de côtes de la France de l'Est dans la première moitié du XXe siècle », Revue Géographique de l'Est [En ligne], vol. 39 / 1 | 1999, mis en ligne le 07 septembre 2013, consulté le 08 septembre 2020. URL : http://journals.openedition.org/rge/4508 ; DOI : https://doi.org/ $10.4000 /$ rge. 4508

Ce document a été généré automatiquement le 8 septembre 2020

Tous droits réservés 


\title{
L'explication du relief de côtes de la France de l'Est dans la première moitié du XXe siècle
}

\author{
The explanation of the scarplands of eastern France in the first half of XXth \\ century \\ Die Erklärung der Schichtstufenlandentwicklung in Ostfrankreich während der \\ ersten Hälfte des XX. Jhs
}

\section{Michel Deshaies}

1 L'est du Bassin parisien offre à l'observation l'un des plus remarquables paysages de côtes européens. Certes, le commandement des côtes est loin d'y atteindre celui du Jura souabe, dans le Sud-Ouest de l'Allemagne. Cependant, c'est incontestablement dans la partie orientale du bassin de Paris que le relief de côtes atteint sa plus grande extension en Europe. Ainsi, du centre du bassin jusqu'aux Vosges, doit-on franchir neuf fronts de côtes qui, pour la plupart, ont entre 100 et $150 \mathrm{~m}$ d'énergie. Rien d'étonnant, dès lors, à ce que cette région ait constitué un champ d'observation privilégié pour les théoriciens de l'explication du relief. En l'espace d'un demi-siècle (1899-1951), trois théories tentant de rendre compte de la genèse du relief de côtes ont été formulées, en s'appuyant tout ou partie sur l'exemple de l'est du Bassin parisien. L'objet de l'article est de rappeler les différences essentielles qui séparent ces théories, et de montrer dans quelle mesure l'évolution des idées scientifiques, et aussi des considérations extrascientifiques, ont pu jouer un rôle dans la façon dont les géomorphologues français et allemands ont expliqué les paysages de côtes de la France de l'Est. En effet, la situation de la région dans une zone d'affrontement majeur entre les puissances européennes n'a pas été sans influence sur l'interprétation privilégiée par les deux écoles géographiques. 


\section{La théorie davisienne}

2 La théorie proposée par W.M. Davis dans son article de 1899, "The drainage of cuestas » constitue la première explication globale et cohérente du relief de côtes. Par bien des aspects cependant, cet article dans lequel il évoque essentiellement les exemples du Jura souabe et du bassin de Londres apparaît comme la conclusion d'un travail entrepris quelques années plus tôt, et dont il a exposé les premiers résultats dans le célèbre article de 1895, «La Seine, la Meuse et la Moselle ». En effet, l'évocation de quelques captures d'affluents de la Marne ou de l'Aisne, et surtout de celle de la Moselle, lui donnait l'occasion de présenter sa classification génétique des cours d'eau. Dans l'article de 1899, il lui suffit de reprendre cette classification, en montrant comment l'évolution du réseau hydrographique dans les structures sédimentaires entraîne le dégagement de reliefs auxquels, pour la première fois, il propose de donner une appellation scientifique : les cuestas. Dans le cas le plus simple, celui de la plaine côtière où affleurent des couches d'inégale résistance doucement inclinées vers la mer, une première génération de cours d'eau s'établit conformément à la pente de la région : Davis les qualifie de conséquents. Au cours de l'encaissement, des affluents installés dans les affleurements de roches tendres vont apparaitre et se développer plus rapidement en exploitant les possibilités d'affouillement plus aisées que dans les roches résistantes. Ces cours d'eau de deuxième génération sont qualifiés de subséquents par Davis. Ce sont eux qui provoquent le dégagement des fronts de cuestas dont la corniche est sapée par une troisième génération de cours d'eau prenant naissance au toit de la couche tendre imperméable et dévalant le talus: ce sont les cours d'eau obséquents coulant à contre-pendage. Pendant cette évolution, l'allongement des cours d'eau subséquents dans les bandes de roches tendres entraîne la capture des cours d'eau conséquents les moins encaissés (fig. 1). Il en résulte une hiérarchisation du réseau hydrographique au profit des cours d'eau coulant dans le contexte structural le plus favorable. 
Fig. 1 : Schéma du développement des côtes selon la théorie de Davis (extrait du Traité de géographie physique de $\mathrm{E}$. de Martonne, 1935)

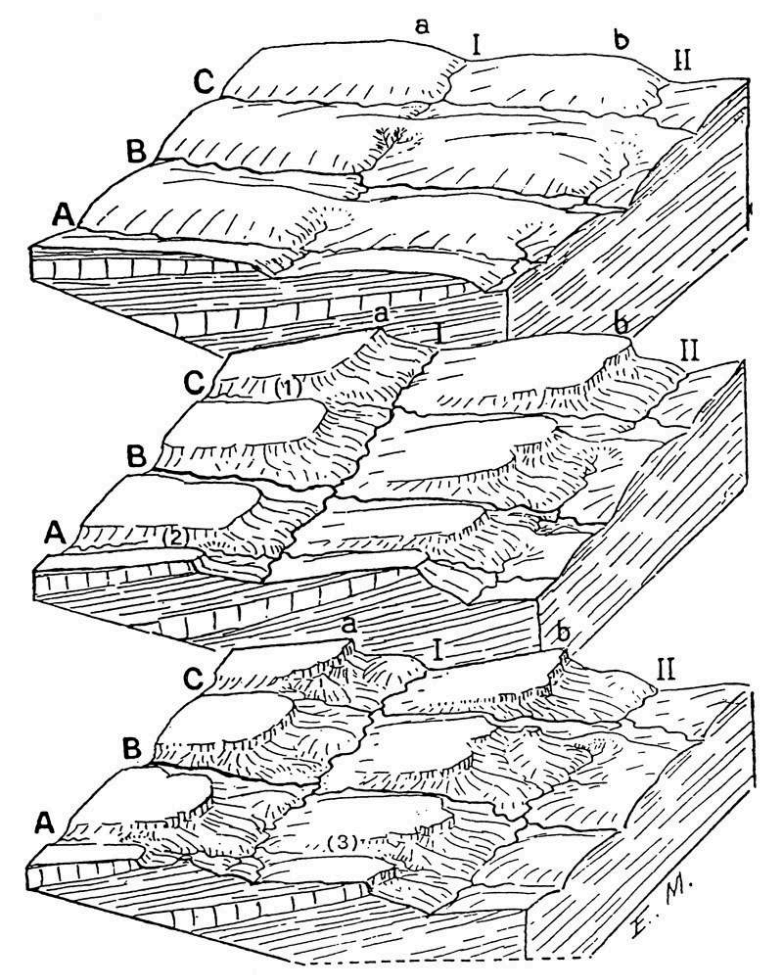

« Notez le recul des côtes (a et b) et leur festonnement de plus en plus marqué. Au deuxième stade, la rivière conséquente $B$ a déjà capturé les cours d'eau $A$ et $C$; les percées conséquentes 1 et 2 sont mortes. Au troisième stade, 1 est un col assez élevé, la percée conséquente 3 est sèche par suite d'une nouvelle capture».

3 L'exemple de la plaine côtière, parfaitement applicable au bassin de Londres, n'est évidemment pas transposable tel quel à l'est du bassin de Paris. Aussi, pour rendre compte du relief de cette région, Davis envisage une évolution en deux phases, ou plutôt deux cycles. Lors d'un cycle antérieur, la région a été réduite à l'état de pénéplaine nivelant aussi bien l'est du bassin sédimentaire que le massif ardennais. C'est sur cette pénéplaine que s'est installé le réseau hydrographique, et notamment la Meuse, dont il explique ainsi le caractère à la fois surimposé et antécédent au soulèvement récent de l'Ardenne. Le relief de cuestas actuel est le résultat du cycle en cours introduit par un nouveau soulèvement d'ensemble de la région.

Il y a évidemment, dans la conception davisienne de l'évolution du relief, un universalisme exprimé par la théorie du cycle d'érosion (1899). Suivant cette théorie, toute portion de l'écorce terrestre soumise à un soulèvement que l'on peut considérer comme rapide, voit son relief progressivement nivelé sous l'action de l'érosion fluviatile, et ce, quelle que soit sa structure. Cette évolution conduit systématiquement au façonnement d'une surface faiblement accidentée nivelant toutes les couches, quelles que soient leur nature et leur structure : la pénéplaine. Dans cette optique, le relief de cuestas, apparu à partir d'une pénéplaine, n'est qu'une phase transitoire et est appelé, à terme, à disparaître.

5 L'explication du relief de côtes par Davis fait l'objet d'un accueil mitigé par les géomorphologues. Certains, comme A. Penck (1899) s'y rallient en appliquant la théorie de Davis au relief de cuestas du Jura souabe. Scheu (1909), puis Reck (1912) proposent à 
leur tour une explication des côtes du Sud-Ouest de l'Allemagne reposant sur la théorie davisienne. En ce qui concerne les reliefs de la France de l'Est, force est de constater que l'explication de Davis est loin de susciter l'enthousiasme des géomorphologues français. Si certains, comme E. de la Margerie ou E. de Lapparent sont des adeptes enthousiastes, d'autres comme E. de Martonne ou Joseph Vidal de la Blache, le fils de l'auteur du "Tableau géographique de la France ", se révèlent beaucoup plus critiques, et, dans un premier temps, se refusent à utiliser le vocabulaire davisien pour la description du relief de la France de l'Est. Ainsi, dans son «étude sur la vallée de la Meuse lorraine ", le capitaine Joseph Vidal de la Blache n'utilise pas une seule fois le terme cuesta proposé par Davis, et s'efforce de montrer que, si la Meuse coule perpendiculairement au pendage des couches du bassin de Paris, elle n'est pas pour autant une rivière subséquente, c'est à dire de deuxième génération comme l'indique Davis dans son article de 1895. En fait, jusqu'à la guerre, aucun auteur ne reprend les termes de Davis pour décrire le relief de la France de l'Est. Il faut attendre 1914 pour voir paraître, dans les Annales de géographie, un article utilisant le vocabulaire davisien : il s'agit de la description du relief de la Vôge par André Cholley, alors jeune professeur de géographie au lycée d'Annecy. À la veille de la guerre, les partisans de la théorie de Davis sont donc encore peu nombreux, et ce, malgré le séjour qu'il a effectué en 1911-1912 en Europe, et au cours duquel il a donné un certain nombre de conférences et mené des excursions sur le terrain qui lui ont permis d'illustrer sa conception.

\section{L'offensive allemande contre l'explication davisienne du relief de côtes}

6 Jusqu'à la guerre, les géomorphologues allemands restent très partagés quant à l'attitude à adopter vis-à-vis des idées de Davis. Le scepticisme d'un certain nombre d'entre eux, parmi les plus notables, comme Alfred Hettner (1913) ou Siegfried Passarge (1912), s'exprime essentiellement sur le concept du cycle d'érosion. En ce qui concerne plus particulièrement l'explication du relief de côtes, la réaction des sceptiques tarde à s'organiser. Ainsi, jusqu'à la guerre, il n'existe pas de théorie concurrente de celle de Davis. Les années qui précèdent la guerre sont au contraire marquées par la diffusion des idées de Davis qui déploie une grande activité pour les faire connaître, notamment en Allemagne, où il donne un cours à l'Université de Berlin durant l'année 1908-1909. De ce séjour à Berlin naîtront plusieurs ouvrages, dont « Die Erklärende Beschreibung der Landformen" (1912), qui est considéré comme étant le véritable traité de géomorphologie davisienne, et dont, malheureusement, il n'existe pas d'équivalent en français, ou même en anglais.

7 L'offensive des géomorphologues allemands contre les idées davisiennes va se déployer après la fin de la guerre. Exprimée globalement contre la théorie du cycle d'érosion dans l'ouvrage posthume de W. Penck (1924), la critique germanique à l'encontre de l'explication davisienne du relief va se développer en particulier sur le relief de côtes qui semble le plus propre à illustrer le caractère trop théorique des idées de Davis. C'est un élève de A. Hettner, le docteur Heinrich Schmitthenner, qui va porter l'offensive et se faire l'un des contradicteurs les plus incisifs de Davis, en proposant une théorie en totale contradiction avec l'explication davisienne du relief de côtes. Esquissée dans sa «Dissertation" portant sur «le façonnement du relief du nord de la Forêt Noire » 
(1913), la théorie de H. Schmitthenner sur le relief de côtes va prendre corps après la guerre dans son étude sur "les formes du relief de côtes entre Meuse et Moselle» (1923).

8 En effet, pendant le premier conflit mondial, de novembre 1915 à novembre 1918, H. Schmitthenner est affecté comme "géologue de guerre » dans la région de Verdun. Il va profiter de ce poste pour faire des observations de terrain extrêmement précises du relief des côtes de Moselle et de Meuse. Par la démarche suivie, comme par le rôle attribué à la structure, et notamment à la tectonique, on peut dire que le travail de Schmitthenner constitue en quelque sorte le négatif de la démarche davisienne et, dans une certaine mesure, l'illustration, appliquée au relief de côtes, des théories de Walther Penck. Par ailleurs, on ne peut manquer de relever le caractère symbolique du terrain d'études, et ce, pour deux raisons : c'est le terrain choisi initialement par Davis (1895) pour développer sa théorie sur le relief de côtes; c'est surtout un terrain où vient de se dérouler le gigantesque affrontement entre l'Allemagne et la France alliée aux AngloSaxons. Dans l'avant-propos du texte de Schmitthenner, on sent poindre l'amertume de la défaite, et dans le corps de l'ouvrage, il y a, dans l'insistance à contredire l'explication davisienne, comme une sorte de volonté de revanche scientifique sur les intuitions anglo-saxonnes.

9 L'ouvrage de Schmitthenner, long de seulement 89 pages, en raison de la situation de pénurie que connaît alors l'Allemagne, commence par une description détaillée (12 pages) du cadre topographique, tectonique et lithologique du champ d'étude: un quadrilatère s'étendant entre Pont-à-Mousson, Saint-Mihiel, Étain et Hagondange, et où se développent les côtes de Meuse, la plaine de la Woëvre et les côtes de Moselle (fig. 2). Suivant une méthode déjà bien rodée par les géomorphologues germaniques, un tiers de l'ouvrage (30 pages) est ensuite consacré à décrire méticuleusement les processus d'érosion en action sur les fronts de côtes, comme sur les plateaux de revers. La dissolution des calcaires par les eaux d'infiltration, les mouvements de masse sur les talus marneux, la formation des sols, l'érosion des sources sont ainsi successivement passés en revue. À chaque fois, l'influence de la lithologie sur l'intensité des processus d'érosion est soulignée. L'apport le plus original de ce chapitre est constitué par l'analyse du développement des vallons élémentaires, drainés dans la Woëvre, et secs sur les hautes surfaces calcaires. À ces vallons qu'il qualifie de "Dellen », et qu'il a pu voir, à maintes reprises, parcourus par un écoulement temporaire entraînant le matériel glissant le long des versants, il attribue un rôle essentiel. Selon lui, leur activité entraîne l'abaissement progressif de la surface, mais dépend étroitement du contexte lithologique rencontré au cours de l'encaissement. Ainsi, dans le chapitre consacré ensuite au développement des vallées, il montre que le nombre de "Dellen " va se modifier avec les progrès de la dénudation. Là où l'ablation d'une couche tendre imperméable laisse apparaître une couche perméable calcaire, une partie des « Dellen " nées sur la couche imperméable va disparaître par infiltration. C'est le cas par exemple sur le revers des côtes de Meuse, là où affleurent les calcaires coralliens de l'Oxfordien. À l'inverse, les nombreuses vallées et «Dellen » de la plaine argileuse de la Woëvre ne sont apparues qu'après l'érosion de la couche de calcaire oxfordien qui la recouvrait. 
Fig. 2 : Tracé des côtes dans l'est du Bassin de Paris d'après H. Schmitthenner (1923)

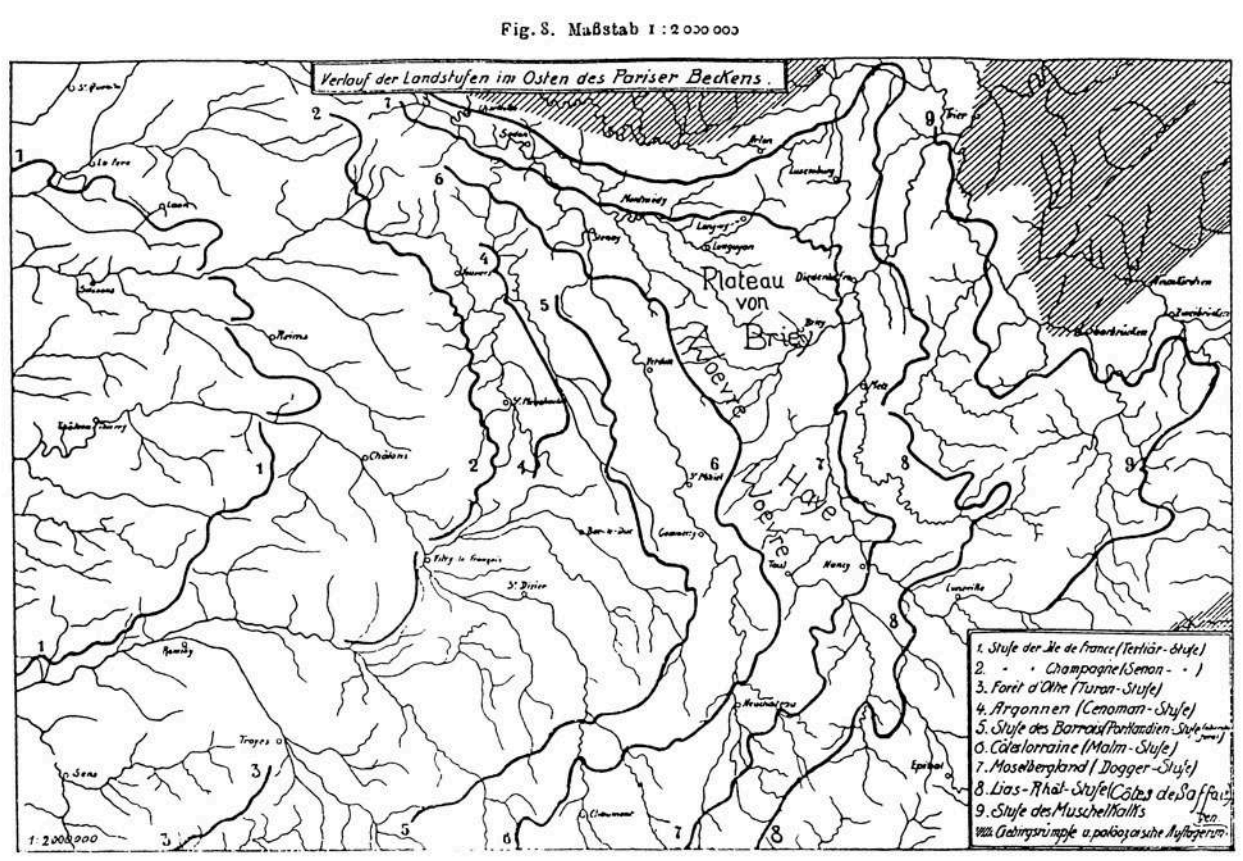

Tout le reste de l'ouvrage (47 pages) consiste, à travers l'explication du développement des vallées, des fronts de côtes et des surfaces de revers, à contester point par point la théorie davisienne. Il lui reste alors, en conclusion, à présenter sa propre théorie de la formation du relief de côtes. Au cœur de l'explication davisienne du relief de côtes, la distinction entre cours d'eau conséquents et subséquents est d'abord vigoureusement contestée.

Ainsi, la question de l'apparition des principaux cours d'eau fournit à Schmitthenner l'occasion d'en découdre franchement avec Davis et sa méthode d'analyse du relief: «La méthode déductive de la morphologie croit pouvoir distinguer des rapports existant entre la direction des cours d'eau et le pendage des couches, des rivières conséquentes ou originelles, des rivières subséquentes, c'est à dire installées ultérieurement. Mais cette déduction ne tient aucun compte de la diversité de la nature. Depuis que la terre est émergée, les forces tectoniques n'ont pas diminué, et il est douteux, voire même invraisemblable, que le pendage des couches soit encore aujourd'hui le même que lors de l'installation des premiers cours d'eau (...). De tous les grands cours d'eau de notre région, aucun ne tient compte du pendage. Ils semblent être tout à fait indépendants de la structure ». Schmitthenner considère donc que, lorsque la Meuse et la pré-Moselle-Meurthe ont établi leur cours, la région était inclinée vers le Nord. C'est en fait «le soulèvement (récent) plus fort de la région orientale qui a fait disparaitre l'ancien pendage tandis que les rivières conservaient leur cours initial ».

Enfin, dans un chapitre consacré au développement des fronts de côtes et des «Landterrassen", autrement dit, des surfaces de revers, il passe en revue la morphologie de détail de ces formes. Dans l'esprit de Schmitthenner, les "Landterrassen », ne sont d'ailleurs pas exactement l'équivalent des surfaces de revers ou "scarplands" au sens où l'entend Davis. Il s'agit en fait de surfaces d'érosion inclinées dans le sens du pendage et recoupant toutes les couches comprises entre deux fronts de côtes. Elles sont donc « strukturbedingt »; c'est à dire que leur inclinaison est 
conditionnée par la structure sans qu'il s'agisse, pour autant, de surfaces structurales. En ce qui concerne le tracé des fronts de côtes, les "Landstufen ", il montre l'influence de la tectonique, et notamment des ondulations anticlinales et synclinales, ainsi que des failles qui déterminent les rentrants, les saillants et la localisation des buttestémoins (Zeugenberg). Aussi, ayant montré que le relief de côtes dépend tyranniquement de la structure, il peut en déduire "qu'une pénéplaine ne se développera jamais dans une région constituée de couches légèrement inclinées, et de résistance et de perméabilité variables ». Il considère la pénéplaine comme une pure construction de l'esprit.

La théorie du relief de côtes présentée par Schmitthenner, en conclusion de son travail, est en quelque sorte l'illustration, appliquée à ce paysage, des théories de W. Penck sur la permanence des mouvements tectoniques. Suivant ce modèle, le soulèvement continu d'un massif ancien et de sa couverture sédimentaire entraîne le dégagement progressif des différents fronts de côtes qui ne sont donc pas tous apparus en même temps (fig 3). Chaque front de côte recule aux dépens d'une "Landterrasse» plus ancienne, tandis que son recul permet l'extension d'une Landterrasse d'âge plus récent. Ainsi, le front de côte le plus proche du massif est aussi le plus récent, et plus on s'éloigne de celui-ci, plus les côtes sont anciennes. Par ailleurs, l'âge d'une même Landterrasse croît vers l'amont pendage. Cette évolution se poursuit tant que dure le soulèvement de la région. Dans cette vision, le recul des côtes, considérable, permet le dégagement progressif des niveaux plus anciens de la série sédimentaire recouvrant le massif, et l'apparition de nouveaux fronts sans que jamais une pénéplaine ne puisse être façonnée. Lorsque le socle lui-même vient à l'affleurement, de nouveaux aplanissements étagés apparaissent, les « Piedmonttreppen » de W. Penck.

Fig. 3 : Développement du relief de côtes et des « Piedmonttreppen » lors du soulèvement d'un massif ancien et de sa couverture sédimentaire d'après Schmitthenner (1941)

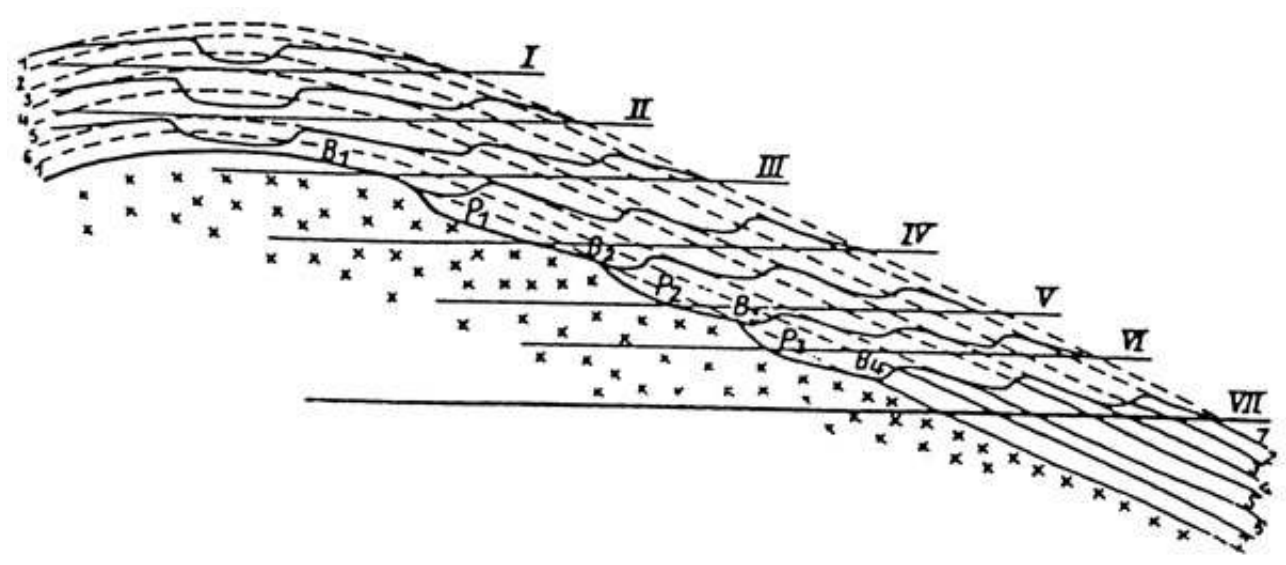

$x x x=$ massif ancien sous la couverture sédimentaire $; 1-7=$ les différents plateaux. Les chiffres impairs désignent les couches tendres; les chiffres pairs les couches dures ; I - VII = les étapes du soulèvement et le relief de côtes correspondant. La ligne horizontale représente le niveau de la mer ; De I à III = développement de trois fronts de côtes (Landstufe) et des Landterrassen correspondantes ; IV = sur le socle mis à nu se développe un premier aplanissement : la «Basislandterrasse B1 »; De V à $\mathrm{VII}=$ relation entre les «Piedmontflächen »P1 à P2 et les « Basislandterrassen » B2 à B4

14 L'ouvrage de Schmitthenner constituait donc, et de loin, l'étude morphostructurale la plus complète qui ait été faite du relief de côtes de l'Est de la France, et beaucoup de ses observations sur la relation entre le tracé des fronts de côtes et la tectonique sont désormais devenues des éléments classiques de la description de cette forme de relief. 
Par ailleurs, sa théorie devenait, par sa cohérence même, une véritable alternative à l'explication proposée par Davis. Rejetant le concept de discontinuité de l'activité tectonique, permettant le façonnement d'une pénéplaine recoupant les couches d'inégale résistance, il proposait au contraire une vision de l'évolution du relief basée sur une sorte de déterminisme structural très éloigné de l'universalisme davisien. Le paysage de côtes, « die Schichtstufenlandschaft » serait ainsi l'expression originale d'un type particulier de structure dont la forme se maintient constamment au cours du temps, et dont il est évidemment exclu de déduire un quelconque stade d'évolution. Pour bien marquer sa différence avec les concepts davisiens, il utilisait un vocabulaire illustrant ses propres concepts : Dellen, Landterrasse, Landstufe.

Par la suite, Schmitthenner ne devait que peu modifier son modèle forgé à partir de l'observation des reliefs de côtes de l'Est de la France. La première modification est la reconnaissance de l'origine périglaciaire des « Dellen » suite notamment aux travaux de J. Büdel $(1938,1957)$ et de J. Tricart $(1949,1951)$. Mais le principal ajout est celui concernant la possibilité, pour les reliefs de côtes, en cours d'évolution, d'être enfouis complètement sous des dépôts (Schmitthenner, 1954, fig. 4). Sur la surface d'aggradation ainsi constituée peut alors s'installer un réseau hydrographique totalement indépendant de la structure; ce qui permettait à Schmitthenner d'expliquer l'inadaptation complète du réseau hydrographique de la France de l'Est.

Fig. 4 : Fossilisation et dégagement d'un relief de côtes d'après Schmitthenner (1954)
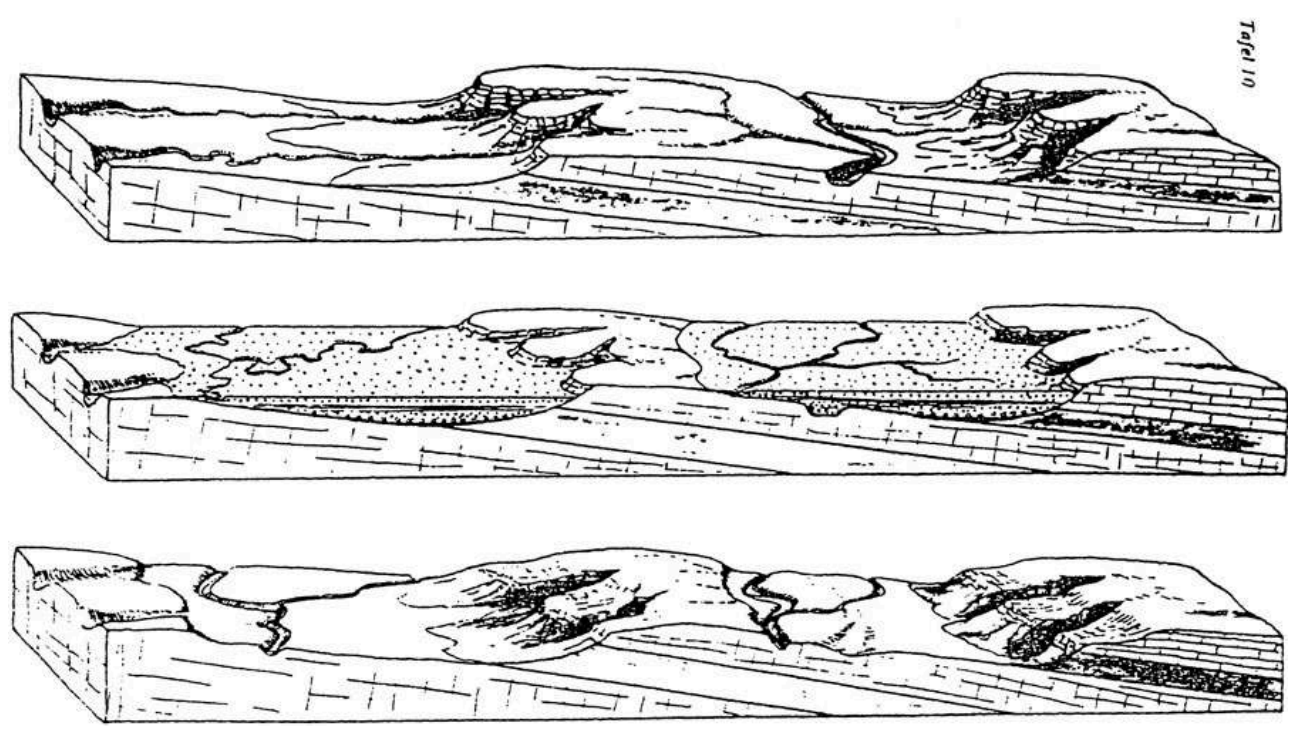

\section{Davis contre Schmitthenner : le choix des écoles nationales}

$16 \mathrm{Au}$ lendemain de la première guerre mondiale on disposait donc, pour le relief de la France de l'Est, de deux explications géomorphologiques en tous points contradictoires. Il se produit alors une divergence entre l'école germanique, qui adopte la théorie de Schmitthenner, et l'école française qui, oubliant les fortes réserves formulées avant la guerre, admet désormais, au moins dans ses grandes lignes, l'explication davisienne. En premier lieu, E. de Martonne, dans les multiples éditions de son célèbre Traité de 
géographie physique, se rallie à la classification génétique des cours d'eau (fig. 1), même si, au fil des éditions, il ajoutera quelques correctifs, signalant "les complications du cycle ». Dans la dixième édition encore (1958), il reprend la théorie de Davis lorsqu'il explique la formation des côtes «en admettant une surface primitive assez inclinée pour orienter la formation du réseau hydrographique, mais sensiblement moins que les surfaces de contact des couches inégalement résistantes qui forment le sous-sol ; Les vallées primitives ou conséquentes forment un réseau d'artères parallèles (...) Les captures se produiront particulièrement le long de la zone où l'évolution des versants aura mis à jour le contact des couches inégalement résistantes. Là se développeront de petits affluents creusant des vallées monoclinales ou subséquentes; ceux de l'artère conséquente la plus vigoureuse capturent les autres; et le cours d'eau subséquent le plus fort est bientôt en état de capter lui-même une tête de vallée conséquente». Certes, la diversité des reliefs de côtes "pourra s'expliquer par la structure », mais ne fera qu'entrainer des «complications du cycle ». Ayant observé que le tracé de la côte des grès du Keuper, dans le bassin de Souabe-Franconie, est compliqué par des ondulations du Trias, il ne mentionne pas pour autant que les côtes lorraines sont aussi affectées par le même type d'ondulations. La seule nuance apportée par de Martonne à la théorie davisienne est la reconnaissance «que les vallées maîtresses se sont établies en Lorraine sous l'influence d'une structure légèrement différente de celle que nous voyons maintenant et se sont enfoncées sur place». Néanmoins, pour de Martonne, le plan incliné descendant des Vosges et recoupant le sommet des côtes qu'avait suggéré le géologue Bleicher (1900), « ne devait pas être autre chose qu'une pénéplaine ».

17 Ce qui est surtout frappant, c'est que la théorie de Davis sur le relief de côtes, non seulement s'impose, mais devient même la seule référence, y compris pour ceux qui tentent parfois de l'aménager à partir d'observations mettant en évidence ses faiblesses. C'est ce que fait, par exemple, R. Capot-Rey (1937), lorsqu'il rédige, dans "Géographie lorraine ", le chapitre consacré au relief. Bien qu'ayant fait un certain nombre d'observations sur l'influence des ondulations anticlinales et synclinales sur le tracé des côtes, il n'en explique pas moins la formation des côtes à partir de la théorie de Davis. La seule inflexion notable est qu'il tente de rendre compte des anomalies que présente le réseau hydrographique, en proposant l'hypothèse d'une surimposition de celui-ci sur une couverture meuble, inclinée du Sud vers le Nord, et masquant indifféremment les couches jurassiques et le Massif ardennais.

18 C'est en fait un peu paradoxalement chez Henri Baulig, l'un des principaux promoteurs des idées de Davis en France, dont il a suivi l'enseignement aux États-Unis (1904-1911), que l'on trouve les aménagements, pour ne pas dire les critiques les plus importantes de la théorie davisienne. Certes, H. Baulig n'a pas écrit d'article portant principalement sur le relief de cuestas. Néanmoins, dans trois de ses plus importants travaux, il évoque suffisamment les paysages de cuestas de l'Est de la France, pour qu'à leur lecture, on saisisse des différences très sensibles avec la théorie de Davis. Les deux premiers sont parus dans les Annales de Géographie et portent sur « le relief de la Haute-Belgique » (1926), et sur « les hauts niveaux d'érosion eustatiques dans le Bassin de Paris » (1928). À la fin du premier article consacré à l'Ardenne, il en vient, bien évidemment, à évoquer, et à essayer d'expliquer le tracé de la Meuse en Lorraine. Ce cours de la Meuse étant "totalement indépendant du tracé des côtes jurassiques", il envisage une «Meuse lorraine, (...) adaptée graduellement à la structure de la nappe crétacée 
transgressive et légèrement discordante ", et se trouvant ainsi "surimposée sur la structure jurassique qui forme seule aujourd'hui les plateaux où elle s'encaisse ». L'érosion des couches crétacées qui masquaient le Jurassique au moment où la Meuse a établi son cours vers le Nord, aurait été stimulée par « la surrection des Vosges relevant les couches secondaires ». On voit immédiatement que cette conception exprimée par $\mathrm{H}$. Baulig rejoint un certain nombre de critiques formulée par $\mathrm{H}$. Schmitthenner à l'encontre de la théorie davisienne. Dans le deuxième article, il montre la fragilité de l'hypothèse de la pénéplaine initiale en montrant l'existence de deux niveaux d'érosion recoupant les points hauts des côtes de Moselle et de Meuse. Celles-ci se maintiennent sur de grandes distances, à des altitudes constantes, atteignant $300 \mathrm{~m}$ pour le niveau d'érosion inférieur, à l'ouest de la Meuse, et $400 \mathrm{~m}$ pour le niveau d'érosion se développant entre Meuse et Moselle. Ces hauts niveaux d'érosion, qu'il attribue aux variations eustatiques, reflètent une évolution complexe du Bassin de Paris, dont les reliefs portent la marque de plusieurs cycles d'érosion, et ont donc subi une importante dénudation.

Dès lors, et c'est l'objet d'un troisième article publié en 1938 (Questions de terminologie), dans le «Journal of Geomorphology », il devient risqué d'appliquer les termes génétiques comme "conséquents", en fonction des relations existant actuellement entre les cours d'eau et le pendage des couches. Ayant rappelé le caractère surimposé du réseau hydrographique de l'est du Bassin de Paris, il conseille d'utiliser plutôt des termes purement descriptifs, comme cataclinal, monoclinal et anaclinal, pour qualifier le tracé des cours d'eau. Est-ce-à-dire que H. Baulig condamne par la même l'explication davisienne du relief de côtes, et notamment celui de la France de l'Est? Pas tout à fait, et il s'en explique nettement dans la suite de l'article en soulignant que si «le schéma théorique de la plaine côtière évoluant d'une manière continue à partir de l'émersion (...) trouvera rarement son application dans la nature, il n'en conserve pas moins sa valeur, d'abord en tant que construction théorique cohérente, irremplacée à ce jour, et ensuite parce que, confronté avec la réalité, il révèle d'ordinaire des discordances qui posent des problèmes et appellent des explications ».

20 En définitive, H. Baulig reprend, avec de sérieux aménagements, l'explication de Davis à l'encontre de laquelle il formule des critiques, qui rejoignent en partie celles émises par les géomorphologues allemands, et notamment Schmitthenner. Pourtant, à aucun moment H. Baulig ne fait référence aux travaux germaniques, dont on peut difficilement supposer qu'il les ait ignorés. Aussi, il est plus vraisemblable de penser que cet ostracisme relève de son scepticisme vis-à-vis des théories de W. Penck; scepticisme tellement fort qu'il conduit à occulter complètement toute autre théorie d'évolution du relief que celle proposée par Davis. En ce qui concerne l'explication du relief de côtes de la France de l'Est, l'occultation de la théorie allemande est totale, puisque l'on n'en trouve pratiquement aucune trace dans les travaux des géomorphologues français s'intéressant à la question. Seul, E. de Martonne (1935), dans son Traité de géographie physique, y fait une courte allusion, mais en en rendant compte très imparfaitement, et pour la condamner très fermement: "Récemment, Schmitthenner a essayé de prouver que les côtes se forment, sans rapport avec l'évolution du drainage superficiel, par le développement de dépressions fermées dans les marnes, qui sont appelées Delle en Lorraine. En réalité, il a simplement montré le rôle que ces phénomènes locaux jouent incontestablement dans le recul des côtes déjà formées; les delles ou mardelles ne peuvent se développer que sur une surface de 
dénudation assez évoluée ». L'ignorance de la théorie allemande est si totale que J. Tricart (1951), dans un article pourtant rédigé en allemand pour la revue Petermann's Mitteilungen, et consacré aux « conditions de développement du relief de côtes dans le Bassin parisien ", affecte de croire qu'il n'existe que la théorie de Davis. Dans toute la critique qu'il formule à l'encontre de la théorie davisienne, au début de l'article, il souligne le rôle de la structure, et notamment des ondulations et des variations d'épaisseur des couches dans les changements de tracé des fronts de côtes, sans jamais faire référence aux travaux de Schmitthenner. Cette méconnaissance lui vaut d'ailleurs une réplique assez vigoureuse, publiée en 1954 dans la même revue, et dans laquelle Schmitthenner rappelle, «Die Regeln der morphologischen Gestaltung im Schichtstufenland », en s'étonnant que J. Tricart « redécouvre une partie des principes qu'il a lui-même déjà mis en évidence il y a trente ans ».

21 Cet ostracisme dont souffre la théorie de Schmitthenner de la part des géomorphologues français contraste fortement avec le crédit que lui accordent, dans le même temps, les géomorphologues allemands. Schmitthenner, qui, entre-temps, a succédé à son maître A. Hettner à l'université de Heidelberg (1928), puis à la direction de la revue Geographische Zeitschrift (1934), est considéré presque unanimement comme le père de ce que J. Büdel (1957) appellera "die klassische Theorie der Schichtstufenlandschaft », en fait, la seule théorie explicative du relief de côtes. Ainsi, dans le grand ouvrage que Robert Gradmann (1931) consacre à l'Allemagne du sud, et où il évoque aussi les côtes lorraines, il n'est question que de la théorie de Schmitthenner, tandis que l'hypothèse de l'existence passée d'une pénéplaine est vigoureusement rejetée. La théorie de Schmitthenner s'impose alors dans l'explication du relief de côtes, jusqu'à ce qu'elle soit de plus en plus fortement critiquée par une nouvelle génération de géomorphologues allemands, comme J. Büdel (1938) ou H. Mortensen (1947 et 1948). Ceux-ci vont mettre en évidence le rôle des paléoclimats dans le façonnement des surfaces d'érosion qui peuvent niveler toutes les couches, quelle que soit leur dureté. On s'achemine alors vers une nouvelle explication du relief de côtes qui, pour l'est du Bassin parisien, va être mise en forme par J. Tricart (1949 et 1951). La principale nouveauté est la reconnaissance de l'empreinte climatique, et la distinction opérée entre des systèmes d'érosion climatique, périglaciaires ou secs, favorables au développement de vastes aplanissements, et des climats, comme le climat tempéré humide, où, au contraire, prédomine l'incision verticale. Dans cette hypothèse, le relief de côtes s'est développé à partir d'un aplanissement façonné sous les climats chauds du Tertiaire. Le dégagement des fronts de côtes résulte de l'incision des vallées sous les climats alternativement tempérés et froids du Quaternaire. Pendant les phases de climats périglaciaires, les surfaces d'érosion des côtes se sont abaissées sous l'action du gel et de l'activité des vallées aujourd'hui sèches, les «Dellen » de Schmitthenner. Lors des phases de climat tempéré humide, l'encaissement des grandes vallées a permis le creusement des dépressions orthoclinales, et donc le dégagement des fronts de côtes, dont le recul est toutefois insignifiant, contrairement à ce que proposait Schmitthenner.

La présentation que fait J. Tricart (1951) de cette nouvelle théorie est quelque peu paradoxale. En effet, il la considère comme étant en contradiction avec l'explication davisienne du relief de côtes. En fait, si les différences avec Davis sont patentes, elles sont plutôt moindres qu'avec la vision de Schmitthenner. J. Tricart reprend finalement l'idée davisienne d'une formation du relief de côtes à partir d'un aplanissement initial 
que Schmitthenner avait rejeté avec vigueur. Seuls les processus responsables de cet aplanissement sont différents.

\section{Conclusion}

Avant la première guerre mondiale, l'explication davisienne du relief de côtes, forgée notamment à partir de l'observation des paysages de l'est du Bassin de Paris, soulève de nombreuses réticences chez les géomorphologues allemands, comme chez les géomorphologues français. Après la guerre, au contraire, on assiste à une divergence complète entre les deux écoles. Alors que la théorie de Davis s'impose largement en France, elle ne trouve plus guère d'écho en Allemagne, où Schmitthenner met au point une théorie opposée, à partir d'observations réalisées pendant la guerre, sur les côtes de Moselle et de Meuse. Or, bien qu'étant issues d'observations réalisées dans la même région, on ne peut pas dire que les deux théories soient en concurrence. C'est plutôt l'ignorance réciproque qui prévaut: on ne trouve nulle trace de la théorie de Schmitthenner dans les travaux des géomorphologues français publiés dans l'entredeux-guerres (et même après d'ailleurs), même si certaines critiques formulées par ceux-ci, à l'encontre de la théorie de Davis, viennent rejoindre celles des géomorphologues allemands. À l'inverse, ceux-ci affectent de croire que la seule explication existant sur la genèse du relief de côtes est celle de Schmitthenner.

Il nous semble qu'une telle divergence d'appréciation, conduisant à ignorer délibérément les arguments de l'adversaire, relèvent de plusieurs considérations. C'est Henri Baulig (1950) qui nous livre l'une d'entre elles, s'agissant du rejet par les géomorphologues allemands de l'explication davisienne: «Au fond, la méthode de Davis, divisant la difficulté, simplifiant délibérément les problèmes, procédant en somme par des approximations successives, répugnait profondément à l'esprit allemand, avide de totalité, peu capable de concevoir la solution partielle d'un problème général ». Ce jugement, somme toute sévère, porté par $\mathrm{H}$. Baulig sur la géomorphologie allemande, nous éclaire en fait aussi sur les raisons du choix opéré par les géomorphologues français en faveur de l'explication davisienne. Entre les deux écoles, il existe une différence non seulement de méthode, mais aussi de nature philosophique. H. Baulig distingue ainsi un esprit allemand, dont cet Alsacien ne partage manifestement pas les valeurs.

Dans quelle mesure les raisons plus simplement nationalistes ont-elles pu jouer dans l'adoption ou le rejet de la théorie anglo-saxonne par les deux écoles de géomorphologues? C'est évidemment plus difficile à évaluer; les savants étant rarement neutres, surtout durant cette période, répugnent néanmoins à avancer ouvertement ce type d'argument. On observera cependant, que les Allemands ont ressenti avec dépit l'engagement américain dans la guerre à partir de 1917. Or, Davis n'est pas resté neutre, puisqu'il a même rédigé un petit manuel destiné à l'usage des soldats américains, et dans lequel était décrit, à l'aide de magnifiques blocsdiagrammes (fig. 5), le relief du Bassin parisien où se déroulaient les opérations militaires (Handbook of Northern France, 1918). Certes, A. Penck continue d'entretenir une correspondance avec Davis, mais les cordiales relations d'avant-guerre sont terminées, et il est assez significatif de voir la critique germanique contre les conceptions davisiennes prendre un singulier développement après la guerre. Celle-ci s'exprime notamment dans l'ouvrage de A. Hettner (1921), «Die Oberflächenformen 
des Festlandes ", ou surtout avec la parution du livre posthume de W. Penck (1924), pour lequel il faudra attendre 1953 avant de disposer d'une traduction en anglais. De plus, il ne faut pas oublier que les géomorphologues allemands, comme A. Hettner, ne se contentent pas d'être des naturalistes. Ce sont aussi des militants de la cause nationaliste allemande, dont une partie importante de l'activité scientifique d'aprèsguerre est consacrée à dénoncer l'injustice faite au peuple allemand par le traité de paix (M. Korinman, 1990). En ce qui concerne les géomorphologues français, on peut également se demander quelle part ont pris les considérations nationalistes dans l'occultation de l'ouvrage de Schmitthenner, qui reste, pendant longtemps, la meilleure étude géomorphologique de ces paysages de côtes, que la France vient alors tout juste d'arracher à l'Allemagne.

Fig. 5 : « La Meuse, la Moselle et la Meurthe dans les quatrième, cinquième et sixième côtes » selon Davis (1918)

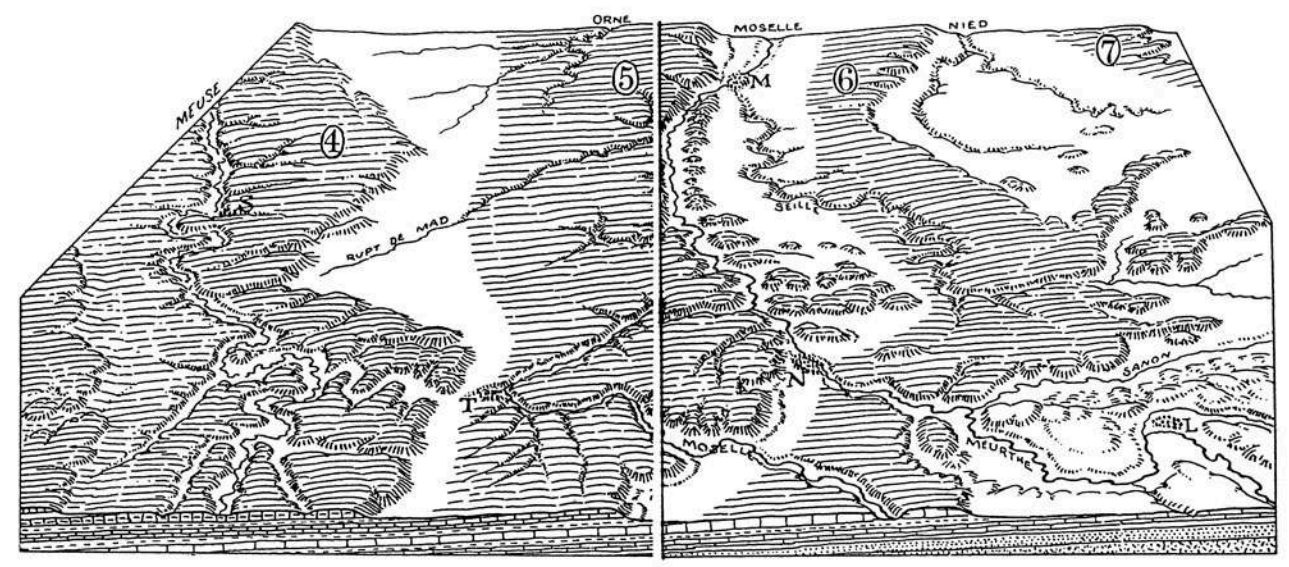

\section{BIBLIOGRAPHIE}

Seuls les ouvrages précédés d'une astérisque sont appelés dans le texte.

*Baulig H. (1926). - Le relief de la Haute-Belgique. Annales de Géographie, n 195, tome XXX, p. 206-235.

*Baulig H. (1928). - Les hauts niveaux d'érosion eustatique dans le Bassin de Paris. Annales de Géographie, t. XXXVII p. 289-305 et 385-406.

*Baulig H. (1938). - Questions de terminologie. Journal of Geomorphology, I, p. 224-229.

*Baulig H. (1950). - Essais de géomorphologie. Publ. de la faculté des Lettres de l'Université de Strasbourg, fasc. 114.

*Bleicher G. (1900). - Observations sur les alluvions anciennes du plateau de Haye. Bull. Soc. Geol. France, t. IX, p. 999.

Blume H. (1971). - Probleme der Schichtstufenlandschaft. Erträge Forschungen, 5 : 117 S.

Darmstadt. 
Blume H. und Wilhelmy H. (1987). - H. Schmitthenner Gedächtnisschrift zu seinem 100 Geburtstag. Erkundliches Wissen, Heft 88.

Bonnefont J.C. et Deshaies M. (1995). - Impact de l'article de Davis : la Seine, la Meuse et la Moselle (1895) sur l'étude des paléoréseaux hydrographiques. R.G.E., t. XXXV, n 3/4, p. 223-234.

*Büdel J. (1938). - Das Verhältnis von Rumpftreppen zu Schichtstufen in ihrer Entwicklung seit dem Alttertiär. Petermanns Geographische Mitteilungen, p. 229-238.

*Büdel J. (1957). - Grundzüge der klimamorphologischen Entwicklung Frankens. Würzburger geogr. Arb., 4/5, S. 5-46.

*Capot-Rey R. (1937). - La structure et le relief, dans Géographie lorraine, p. 41-107.

*Cholley A. (1914). - La Vôge. Annales de Géographie, t. XXIII, n 129, mai, p. 219-225.

Chorley, Beckinsale, Dunn (1973). - The history of the study of landforms, Methuen, vol. 2, $874 \mathrm{p}$.

*Davis W.M. (1895). - La Seine, la Meuse et la Moselle. Annales de Géographie, t. V, n 19, 15 octobre, p. 25-49, 1 pl. h.t.

*Davis W.M. (1899). - The drainage of cuestas. Proc. Geologist's Assoc., XVI, part II, may, p. 75-93, 16 fig.

Davis W. M. (1899). - La pénéplaine. Annales de Géographie, t. VIII, $n^{\circ}$ 40, p. 289 - 303, et n 42, p. 385-404.

Davis W.M. (1909). - Geographical Essays. edit. by D.W. Johnson, Ginn and Company, Boston.

Davis W.M. und Braun G. (1911). - Grundzüge der Physiogeographie. Leipzig und Berlin, 322 p.

*Davis W.M. (1912). - Die erklärende Beschreibung der Landformen, B.G. Teubner, Leipzig, 564 p.

*Davis W.M. (1918). - Handbook of Northern France, 169 p.

Derruau M. (1994). - The quintessial systematist : H. Baulig. Geomorphology 11, p. 1-13.

Gradmann R. (1919). - Das Schichtstufenland. Z.G.E., nº 3-4.

*Gradmann R. (1931). - Süddeutschland, Band 2, die einzelnen Landschaften. J. Engelhorn, Stuttgart, $553 \mathrm{p}$.

*Hettner A. (1913). - Die Entstehung der Täler. Geographische Zeitschrift, S. 155.

*Hettner A. (1921). - Die Oberflächenformen des Festlandes. B.G. Teubner, Leipzig.

*Korinman M. (1990). - Quand l'Allemagne pensait le monde. Fayard, Paris, 412 p.

Joly H. (1911). - Géographie physique de la Lorraine et de ses enveloppes. A. Barbier éditeur, Nancy, $350 \mathrm{p}$.

Lapparent (de) A. (1896). - Leçons de géographie physique. Paris, Masson et Cie, in $8^{\circ}$, XVI, 590 p.

Martonne (de) E. (1898). - Problèmes de l'histoire des vallées, Enns, Salzach. Annales de Géographie, t. VII, nº 36, p. 385-403.

Martonne (de) E. (1899). - Une excursion de géographie physique dans le Morvan et l'Auxois. Annales de Géographie, t. VIII, n 42, p. 405-419.

*Martonne (de) E. (10 éditions de 1909 à 1958). - Traité de géographie physique, vol. 2, le relief du sol. Martonne (de) E. (1942). - France physique, in Géographie Universelle, A. Colin, Paris.

*Mortensen H. (1948). - Das Verhältnis von Rumpftreppen zu Schichtstufen in ihrer Entwicklung seit dem Alttertiär. Petermanns Geographische Mitteilungen, S. 1-13. 
*Passarge S. (1912). - Physiologische Morphologie. Mitteilungen der Geographischen Gesellschaft in Hamburg, Vol. 26, S. 133-337.

*Penck A. (1899). - Talgeschichte der obersten Donau. Schriften des Vereins für Geschichte des Bodensees und seiner Umgebung, t 28, S. 117-130.

*Penck W. (1924). - Die morphologische Analyse, Stuttgart.

Penck W. (1953). - Morphological analysis of lanforms. Macmill and Co, London, 429 p.

*Reck H. (1912). - Die morphologische Entwicklung der süddeutschen Schichtstufenland im Lichte der Davisschen Zyklustheorie. Zeitschr. der Deutschen Geol. Gesellsch. B. 64, 81 S.

*Scheu E. (1909). - Zur Morphologie der schwäbisch-fränkischen Stufenlandschaft. Forschungen zur Deutschen Landes-und Volkskunde, XVIII, 4, p. 365-403.

*Schmitthenner H. (1913). - Die Oberflächengestaltung des nördlichen Schwarzwaldes. Abhandlungen zur badischen Landeskunde, Karlsruhe, $109 \mathrm{~S}$.

Schmitthenner H. (1920). - Die Entstehung der Stufenlandschaft. Geogr. Zeitschr., 207-209.

*Schmitthenner H. (1923). - Die Oberflächenformen der Stufenlandschaft zwischen Maas und Mosel. Verlag von J. Engelhorn Nachf., Stuttgart, $89 \mathrm{~S}$.

Schmitthenner H. (1925). - Die Entstehung der Dellen und ihre morphologische Bedeutung. Zeitschrift für Geomorphologie I.

Schmitthenner H. (1930). - Das Problem der Stufenlandschaft. Petermanns Geographische Mitteilungen, Erg. Heft 209, S. 97-109.

*Schmitthenner H. (1954). - Die Regeln der morphologischen Gestaltung im Schichtstufenland. Petermanns Geographische Mitteilungen, 98, S. 3-10.

Schmitthenner H. (1956). - Probleme der Schichtstufenlandschaft. Marburger Geographische Schriften, t. 3, 87 S.

Supan E. (1903). - Grundzüge der Physischen Erdkunde. Leipzig Verlag von Veit et Comp., 852 p.

*Tricart J. (1949). - La partie orientale du Bassin de Paris. Étude morphologique. Thèse, Paris, S.E.D.E.S. éd. t. 1 : La genèse du bassin, t. 2 : l'évolution morphologique.

*Tricart J. (1951). - Die Entstehungsbedingungen des Schichtstufenreliefs im Pariser becken, Petermanns Geographische Mitteilungen, S. 98-105.

Vidal de la Blache J. (1908). - Étude sur la vallée lorraine de la Meuse. A. Colin éd., Paris, 183 p.

\section{RÉSUMÉS}

Dans la première moitié du XXe siècle, deux théories opposées tentent de rendre compte de la genèse du relief de côtes dans la France de l'Est. L'explication proposée par W. M. Davis (1899) part de l'hypothèse d'une surface plane initiale, à partir de laquelle l'encaissement des cours d'eau permet le dégagement des fronts de côtes. Après avoir, dans un premier temps, suscité beaucoup de critiques, elle est néanmoins adoptée, après le premier conflit mondial, par la majorité des géomorphologues français. À l'inverse, les géomorphologues allemands s'éloignent de la théorie davisienne, pour adopter l'explication proposée par H. Schmitthenner en 1923. Celui-ci rejette l'hypothèse de la pénéplaine, et considère que le relief de côtes est la seule forme de relief pouvant se développer sur une série sédimentaire faiblement inclinée, où alternent des roches d'inégale résistance. Des différences fondamentales de conception de l'évolution du relief, 
autant que des motivations plus prosaïquement nationalistes expliquent les choix faits par les Écoles nationales. Après la Seconde guerre mondiale, le renouvellement de l'explication du relief de côtes se fait à travers les travaux d'une nouvelle génération de géomorphologues allemands qui, en mettant en évidence l'importance des facteurs climatiques, reviennent à la conception de l'existence d'un aplanissement initial, appelé désormais surface d'érosion.

In the first half of the XXth century two opposing theories were proposed to explain the genesis of cuesta landscape in eastern France. According to Davis (1899), incision of a river network into a peneplain involves the development of scarps. This Davisian's theory is at first criticized, but, after the First World War it is acknowledged by French geomorphologists. On the other hand, German geographers turn away from Davis's theory in favour of Schmitthenner's explanation (1923). Schmitthenner rejects the peneplain hypothesis and considers that scarpland is the only form of relief which can be developed on a gently inclined series of strata of unequal resistance. These fundamental conceptual differences concerning landscape evolution as much as more prosaic nationalistic motivations explain the preferences of national Schools. After the Second World War, the explanation of scarpland is reopened by the work of a new generation of German geomorphologists, which underlines the importance of climatic factors, reverting to the idea of an initial plane surface : from now on called an erosion surface.

Während der ersten Hälfte des XX. Jhs., wird die Genese der Schichtstufenlandschaft in Ostfrankreich durch zwei gegensätzliche Theorien erklärt. Die von Davis (1899) vorgeschlagene Erklärung geht von der Hypothese einer ebenen Ursprungsfläche aus, aus der durch die Aktion von Flußläufen Schichtstufen freigelegt werden. Nachdem sie anfänglich viel kritisiert wurde, gilt sie jedoch, nach dem ersten Weltkrieg, als von der Mehrheit der französischen Geomorphologen angenommen. Im Gegenzug entfernen sich die deutschen Geomorphologen von der Theorie Davis' und folgen der von Schmitthenner 1923 vorgeschlagenen Erklärung. Besonders die Annahme der Ausgangsfläche, der sogenannten «Peneplain » wird von Schmitthenner widerlegt. Er betrachtet die Schichtstufenlandschaft als die einzige Reliefform, die sich aus schwach geneigten Schichten, in denen unterschiedlich feste Steinschichten alternieren, bilden kann. Grundlegende Unterschiede in der Auffassung von der Reliefbildung, wie auch nationalistische Beweggründe, erklären die jeweiligen Deutungen der beiden Nationen. Nach dem zweiten Weltkrieg wird die Erklärung der Schichtstufenlandschaft durch die Arbeiten einer neuen deutschen Geomorphologengeneration wieder aufgenommen. Sie hat die Auffassung einer Ausgangsfläche, einer nunmehr sogenannten « Rumpffläche », erneuert und dabei die Rolle klimabedingter Prozesse unterstrichen.

\section{INDEX}

Mots-clés : Davis, pénéplaine, relief de côtes, Schmitthenner

Schlüsselwörter : Davis, Peneplain, Schichtstufenland, Schmitthenner

Keywords : Davis, peneplain, scarpland, Schmitthenner

\section{AUTEUR}

\section{MICHEL DESHAIES}

Département de géographie - Université de Nancy 2 - BP 339754015 Nancy Cedex 